\title{
Úlcera venosa: caracterização dos atendimentos em ambulatório de hospital universitário
}

\section{Venous ulcer: characterization of outpatient care at a university hospital Úlcera venosa: caracterización de la atención ambulatoria en un hospital universitário}

Aline Silva de Oliveira ${ }^{1 *}$, Débora Lira Correia², Karla Vanessa Pinto Vasconcelos², Saionara Leal Ferreira ${ }^{3}$, Francisca Alexandra Araújo da Silva³, Solange Gurgel Alexandre ${ }^{3}$

ORCID IDS

Oliveira AS (D) https://orcid.org/0000-0003-0799-1719

Correia DL (D) https://orcid.org/0000-0001-5435-7759

Vasconcelos KVP (D) https://orcid.org/0000-0001-7851-4042

Ferreira SL (D) https://orcid.org/0000-0002-4131-7077

Silva FAA (D) https://orcid.org/0000-0001-6823-0193

Alexandre SG (D) https://orcid.org/0000-0001-6742-1043

\section{COMO CITAR}

Oliveira AS; Correia DL; Vasconcelos KVP; Ferreira SL; Silva FAA; Alexandre SG. Úlcera venosa: caracterização dos atendimentos em ambulatório de hospital universitário. ESTIMA, Braz. J. Enterostomal Ther., 2020, 18: e2320. https://doi.org/10.30886/estima.v18.928_PT

\section{RESUMO}

Objetivo: caracterizar o perfil clínico e sociodemográfico de pessoas com úlceras venosas acompanhadas em ambulatório especializado de um hospital universitário. Método: estudo documental realizado em ambulatório de cirurgia geral de um hospital universitário. Realizou-se a revisão de 104 instrumentos aplicados na primeira consulta de enfermagem. Resultados: houve predominância do sexo feminino, com média de idade de 54 anos. A maioria não exercia atividade remunerada, sendo a ferida o motivo do afastamento das atividades laborais. A hipertensão arterial sistêmica e a obesidade foram as comorbidades mais prevalentes. A pessoa com úlcera venosa foi a principal envolvida no cuidado direto com a ferida. Conclusão: o perfil da população do estudo se mostra compatível com outros estudos em diferentes contextos do Brasil. Os resultados permitem uma reflexão acerca da assistência prestada e efetividade das intervenções implementadas.

DESCRITORES: Úlcera varicosa; Epidemiologia; Perfil de saúde; Estomaterapia.

\footnotetext{
1. Universidade Estadual do Ceará - Departamento de Enfermagem - Fortaleza (CE), Brasil.

2. Universidade Federal do Ceará - Departamento de Enfermagem - Fortaleza (CE), Brasil.

3. Hospital Universitário Walter Cantídio - Ambulatório de Cirurgia - Fortaleza (CE), Brasil.

*Autora correspondente: alinesoliveira312@gmail.com

Recebido: Jul. 25, 2020 | Aceito: Set. 22, 2020
} 


\begin{abstract}
Objective: To characterize the clinical and sociodemographic profile of people with venous ulcers followed up in a specialized outpatient clinic of a university hospital. Method: documentary study carried out in a general surgery outpatient clinic of a university hospital. 104 instruments applied in the first nursing consultation were reviewed. Results: there was a predominance of females, with a mean age of 54 years. The majority did not perform paid work, the wound being the reason for leaving work activities. Systemic arterial hypertension and obesity were the most prevalent comorbidities. The person with venous ulcer was the main person involved in direct care of the wound. Conclusion: the profile of the study population is compatible with other studies in different contexts in Brazil. The results allow a reflection on the assistance provided and the effectiveness of the implemented interventions.
\end{abstract}

DESCRIPTORS: Varicose ulcer; Epidemiology; Health profile; Stomatherapy.

\title{
RESUMEN
}

Objetivo: caracterizar el perfil clínico y sociodemográfico de las personas con úlceras venosas seguidas en una consulta externa especializada de un hospital universitario. Métodos: estudio documental realizado en una consulta externa de cirugía general de un hospital universitario. Se revisaron 104 instrumentos aplicados en la primera consulta de enfermería. Resultados: Predominó el sexo femenino, con una edad media de 54 años. La mayoría no realizó trabajo remunerado, siendo la herida la razón por la que dejó las actividades laborales. La hipertensión arterial sistémica y la obesidad fueron las comorbilidades más prevalentes. La persona con úlcera venosa fue la persona principal involucrada en el cuidado directo de la herida. Conclusión: El perfil de la población de estudio es compatible con otros estudios en diferentes contextos en Brasil. Los resultados permiten reflexionar sobre la asistencia brindada y la efectividad de las intervenciones implementadas.

DESCRIPTORES: Úlcera Varicosa; Epidemiología; Perfil de Salud; Estomaterapia.

\section{INTRODUÇÃO}

A úlcera de etiologia venosa é a mais comum das úlceras de membros inferiores, correspondendo a aproximadamente 80 a $90 \%$ destas feridas ${ }^{1}$. É definida como uma área de descontinuidade da epiderme que persiste por quatro semanas ou mais e ocorre como resultado da hipertensão venosa e insuficiência da bomba do músculo gastrocnêmio ${ }^{2}$.

As feridas surgem de forma espontânea ou traumática, sendo a localização mais frequente a região maleolar e o terço distal da perna. Podem ser únicas ou múltiplas e de tamanhos variados. Geralmente são superficiais, muito exsudativas, podendo ser dolorosas, aspecto que melhora com a elevação dos membros ${ }^{3}$.

A úlcera venosa (UV) é uma condição de saúde antiga e um importante problema de saúde pública devido ao impacto negativo na qualidade de vida da pessoa com a ferida e ao decorrente aumento nos gastos com saúde a nível individual e governamental. As atividades da vida diária podem ficar comprometidas devido à dor, dificuldade de mobilidade e diminuição da produtividade. Essa, muitas vezes, resulta em afastamento do ambiente de trabalho e aposentadorias por invalidez em pessoas em idade produtiva. As relações sociais também podem ser afetadas, sobretudo em indivíduos cujas feridas apresentam exsudação abundante e odor, resultando em isolamento social, baixa autoestima e sentimento de desesperança com relação ao tratamento ${ }^{4-6}$.

Devido à complexidade das feridas, seu lento processo de cicatrização e consequente impacto na qualidade de vida, a assistência à pessoa com UV deve ser individualizada e integral. Os cuidados relativos à cicatrização da ferida devem ser realizados por profissional qualificado que utilize raciocínio e julgamento clínico para escolha do tratamento adequado e acompanhamento do caso, sendo o enfermeiro estomaterapeuta o profissional de destaque para a prática, uma vez que possui formação diferenciada na área de feridas.

A Estomaterapia é uma especialidade exclusiva do enfermeiro, voltada para o cuidado de pessoas com estomias, feridas e incontinências, nos seus aspectos preventivos, terapêuticos e de reabilitação com vistas à melhoria da qualidade de vida ${ }^{7}$. A assistência do enfermeiro estomaterapeuta é realizada por meio da consulta de enfermagem norteada pela Sistematização da Assistência de Enfermagem (SAE).

A consulta de enfermagem direcionada à pessoa com ferida crônica configura-se como uma tecnologia levedura de grande relevância para o êxito do tratamento, pois se mostra eficaz para a deteç̧ão dos requisitos do 
autocuidado, assim como o acompanhamento das medidas instituídas de forma individualizada e apropriada às necessidades específicas de cada pessoa. Permite, ainda, que sejam desenvolvidas habilidades próprias para a melhora de sua qualidade de vida ${ }^{8}$.

Devido à indisponibilidade de uma estimativa oficial relativa à prevalência e incidência de UV, seu perfil na população brasileira, assim como a assistência de enfermagem prestada a essas pessoas, surgiu o seguinte questionamento: qual o perfil clínico e sociodemográfico de pessoas com UVs acompanhadas em ambulatório especializado de um hospital universitário?

\section{OBJETIVO}

Caracterizar o perfil clínico e sociodemográfico de pessoas com UV acompanhadas em ambulatório especializado de um hospital universitário.

\section{MÉTODOS}

Estudo documental realizado em um ambulatório especializado de um hospital universitário do município de Fortaleza-Ceará, que atende, dentre outros, pessoas com UVs.

A população do estudo foi composta por prontuários de pessoas com UVs, maiores de 18 anos, de ambos os sexos, que estiveram em acompanhamento no período de 2015 a 2018, no referido ambulatório.

Utilizou-se como critério de inclusão: pessoa com UV que tenha realizado a primeira consulta de enfermagem com preenchimento de instrumento utilizado no serviço como parte da SAE. Foram excluídas pessoas com úlceras de outras etiologias, como arteriais ou mistas (venosa e arterial).

Foi identificado o total de 312 pessoas com feridas, acompanhadas no referido ambulatório, sendo localizados 184 instrumentos utilizados nas primeiras consultas de enfermagem. Desses, 104 pertenciam a pessoas com diagnósticos de UV, que atenderam aos critérios de inclusão, totalizando a amostra do estudo, sendo incluídas para análise.

$\mathrm{O}$ estudo foi realizado seguindo as recomendações éticas da resolução 466/2012 do Conselho Nacional de Saúde. A pesquisa foi aprovada pelo Comitê de Ética em
Pesquisa da Universidade Federal do Ceará, sob parecer 699.599 e CAAE 85767218.1.0000.5045.

\section{RESULTADOS}

A maioria dos participantes da pesquisa era do sexo feminino $(57,7 \% ; 60)$. A idade variou de 24 a 87 anos, com média de $58 \pm 14$ anos de idade. Predominaram indivíduos casados ou em união estável (44,2\%; 46), com ensino fundamental $(53,8 \% ; 56)$ e renda familiar de um a dois salários mínimos $(64,4 \% ; 67)$ (Tabela 1).

Cerca de 76\% (80) dos participantes da pesquisa estavam sem exercer atividade remunerada no momento do atendimento, sendo a ferida o motivo para o afastamento das atividades laborais em 46,2\% (37) dos casos. Foi verificado que $34,6 \%$ (36) recebiam benefício previdenciário devido à existência da UV.

Foi verificado o registro de comorbidades em $49(47,1 \%)$ prontuários. A hipertensão arterial sistêmica (HAS) e a obesidade foram as mais prevalentes, respectivamente em $39,4 \%$ (41) e 18,3\% (19) dos participantes. Foi verificada a presença de duas comorbidades em $18,3 \%$ (19) e a presença de três ou mais comorbidades em 15,4\% (16), sendo a associação mais comum HAS e diabetes mellitus $(10,6 \% ; 11)$ (Tabela 2).

Quanto ao uso de medicamentos, $67,3 \%$ (70) faziam uso de algum tipo de medicamento no momento da consulta. As classes de medicamentos mais utilizados foram os antihipertensivos com $25 \%$ (26) e analgésicos e antivaricosos com $17,3 \%$ (18) cada.

A maioria dos indivíduos apresentava uma única úlcera $(78,8 \% ; 82)$, localizada predominantemente na região maleolar (33,6\%; 35). A área das lesões variou de 0,25 a $510 \mathrm{~cm}^{2}$, com mediana de $17,1 \mathrm{~cm}^{2}$ e intervalo interquartil de 5 a $44 \mathrm{~cm}^{2}$, sendo a maioria com área de lesão maior que $20 \mathrm{~cm}^{2}$ (31,7\%; 33). O tempo de existência da UV variou de 1 mês a 46 anos, sendo mais comum as lesões com mais de 10 anos de existência $(31,7 \% ; 33)$ (Tabela 3).

Mais de 92\% (96) das pessoas com UV realizavam a troca do curativo no domicílio, utilizando principalmente soro fisiológico $(77,0 \% ; 74)$ para a limpeza da ferida, seguido de água e sabão (11,5\%; 11); nos demais prontuários (11,5\%; 11) não havia registro acerca do produto utilizado para a limpeza da ferida. 
Tabela 1. Características sociodemográficas de pessoas com úlcera venosa em atendimento ambulatorial em hospital universitário. Fortaleza (CE), Brasil - 2015 a 2018.

\begin{tabular}{|c|c|}
\hline Sexo & n (\%) \\
\hline Feminino & $60(57,7)$ \\
\hline Masculino & $44(42,3)$ \\
\hline Estado civil & n (\%) \\
\hline Casado/União estável & $46(44,2)$ \\
\hline Separado & $5(4,8)$ \\
\hline Solteiro & $35(33,7)$ \\
\hline Viúvo & $18(17,3)$ \\
\hline Faixa etária & n (\%) \\
\hline 24 a 39 anos & $9(8,7)$ \\
\hline 40 a 59 anos & $48(46,1)$ \\
\hline 60 anos ou mais & $47(45,2)$ \\
\hline Escolaridade & $\mathrm{n}(\%)$ \\
\hline $\begin{array}{c}\text { Não alfabetizado/ } \\
\text { Analfabetismo funcional }\end{array}$ & $16(15,4)$ \\
\hline Ensino fundamental & $56(53,8)$ \\
\hline Ensino médio & $24(23,0)$ \\
\hline Ensino superior & $3(3,0)$ \\
\hline Sem registro & $5(4,8)$ \\
\hline Renda familiar & n (\%) \\
\hline Até um salário mínimo & $24(23,1)$ \\
\hline Um a dois salários mínimos & $67(64,4)$ \\
\hline Três a quatro salários mínimos & $10(9,6)$ \\
\hline Sem registro & $3(2,9)$ \\
\hline $\begin{array}{l}\text { Exercício de atividade } \\
\text { remunerada }\end{array}$ & n (\%) \\
\hline $\operatorname{sim}$ & $24(23,1)$ \\
\hline Não & $80(76,9)$ \\
\hline
\end{tabular}

Tabela 2. Comorbidades e medicamentos utilizados por pessoas com úlcera venosa em atendimento ambulatorial em hospital universitário. Fortaleza (CE), Brasil - 2015 a 2018.

\begin{tabular}{|c|c|}
\hline Comorbidades & n (\%) \\
\hline Hipertensão arterial sistêmica & $41(39,4)$ \\
\hline Obesidade & $19(18,3)$ \\
\hline Diabetes Mellitus & $17(16,3)$ \\
\hline Etilismo & $13(12,5)$ \\
\hline Cardiopatia & $10(9,6)$ \\
\hline Dislipidemia & $8(7,7)$ \\
\hline Nefropatia & $4(3,8)$ \\
\hline Distúrbios de coagulação & $4(3,8)$ \\
\hline Outras* & $9(8,6)$ \\
\hline Sem registro & $55(52,9)$ \\
\hline Medicamentos & n (\%) \\
\hline Anti-hipertensivos & $26(25,0)$ \\
\hline Analgésicos & $18(17,3)$ \\
\hline Antivaricosos & $18(17,3)$ \\
\hline Antibióticos & $9(6,7)$ \\
\hline Anti-inflamatórios & $9(6,7)$ \\
\hline Hipoglicemiante & $7(8,6)$ \\
\hline Hipolipemiante & $7(8,6)$ \\
\hline $\begin{array}{l}\text { Anticoagulantes/ } \\
\text { Antiagregante plaquetário }\end{array}$ & $4(3,8)$ \\
\hline Outros** & $27(26,0)$ \\
\hline Sem registro & $34(32,7)$ \\
\hline
\end{tabular}

*Osteoporose (3), artrite reumatoide (1), transplante renal (1), hipotireoidismo (1), mioma uterino (1); **Diuréticos, antiulcerativos, glicocorticóide, imunossupressor. 
Tabela 3. Características das úlceras venosas em pessoas em atendimento ambulatorial em hospital universitário. Fortaleza (CE), Brasil - 2015 a 2018.

\begin{tabular}{|c|c|}
\hline Quantidade de úlceras & $\mathrm{n}(\%)$ \\
\hline Uma & $82(78,8)$ \\
\hline Duas & $17(16,3)$ \\
\hline Três & $3(2,9)$ \\
\hline Quatro & $2(2,0)$ \\
\hline Localização das úlceras & $\mathrm{n}(\%)$ \\
\hline Maléolo & $26(25,0)$ \\
\hline Terço distal da perna & $18(17,3)$ \\
\hline Registro apenas do MID ou MIE & $18(17,3)$ \\
\hline Terço médio da perna & $9(6,7)$ \\
\hline Pé & $9(6,7)$ \\
\hline Terço medial + distal & $7(8,6)$ \\
\hline Terço distal + pé & $2(1,9)$ \\
\hline Maléolo + pé & $2(1,9)$ \\
\hline Terço proximal da perna & $1(1,0)$ \\
\hline Calcâneo & $1(1,0)$ \\
\hline Sem registro & $8(7,7)$ \\
\hline Área da úlcera & $\mathrm{n}(\%)$ \\
\hline Menor que $5 \mathrm{~cm}^{2}$ & $17(16,4)$ \\
\hline$\geq 5$ a $10 \mathrm{~cm}^{2}$ & $10(9,6)$ \\
\hline$\geq 10$ a $15 \mathrm{~cm}^{2}$ & $5(4,8)$ \\
\hline$\geq 15$ a $20 \mathrm{~cm}^{2}$ & $6(5,8)$ \\
\hline Maior que $20 \mathrm{~cm}^{2}$ & $33(31,7)$ \\
\hline Sem registro & $33(31,7)$ \\
\hline Tempo de úlcera & $\mathrm{n}(\%)$ \\
\hline Menor de 1 ano & $18(17,3)$ \\
\hline$\geq 1$ a 5 anos & $25(24,0)$ \\
\hline
\end{tabular}

continua...
Tabela 3. Continuação...

\begin{tabular}{|c|c|}
\hline Tempo de úlcera & $\mathrm{n}(\%)$ \\
\hline$\geq 5$ a 10 anos & $21(20,2)$ \\
\hline$\geq 10$ anos & $33(31,7)$ \\
\hline Sem registro & $7(6,8)$ \\
\hline $\begin{array}{l}\text { Coberturas utilizadas } \\
\text { a nível ambulatorial }\end{array}$ & $\mathrm{n}(\%)$ \\
\hline Bota de Unna & $69(66,3)$ \\
\hline Carvão ativado com prata & $7(6,7)$ \\
\hline Colagenase & $7(6,7)$ \\
\hline $\begin{array}{c}\text { Óleo a base de ácidos } \\
\text { graxos essenciais }\end{array}$ & $6(5,9)$ \\
\hline Hidrofibra com prata & $5(4,8)$ \\
\hline Hidrogel & $5(4,8)$ \\
\hline Alginato de cálcio & $2(1,9)$ \\
\hline Hidrocolóide & $2(1,9)$ \\
\hline PHMB gel & $1(1,0)$ \\
\hline
\end{tabular}

$\mathrm{MID}=$ membro inferior direito; $\mathrm{MIE}=$ membro inferior esquerdo; $\mathrm{PHMB}$ $=$ polihexanida

Dentre as pessoas investigadas, apenas nove relataram o uso de alguma cobertura primária para a realização do curativo no domicílio. Os produtos citados foram: ácidos graxos essenciais (AGE), colagenase, pomada cicatrizante à base de barbatimão, sulfadiazina de prata e óleo mineral. Apenas $36(34,6 \%)$ informaram aderir à terapia compressiva, utilizando faixa elástica ou meias de compressão.

Em âmbito ambulatorial, a bota de Unna, terapia compressiva inelástica, foi a cobertura mais utilizada para o tratamento das lesões $(66,3 \% ; 69)$, seguido de carvão ativado com prata $(6,7 \% ; 7)$, colagenase $(6,7 \% ; 7)$ e óleo à base de AGE (5,9\%; 6) (Tabela 3).

Não foi encontrado preenchimento da cobertura em quatro instrumentos. Porém foi contabilizado o uso de mais de 100 produtos, devido à associação de alguns deles, como óleo a base de AGE e hidrogel, óleo a base de AGE e 
colagenase, carvão ativado com prata e Polihexanida (PHMB) gel, bota de Unna e carvão ativado com prata.

Em relação ao envolvimento nos cuidados com a ferida a nível domiciliar, foram encontrados registros em 95 instrumentos, sendo a própria pessoa com UV a principal envolvida no cuidado direto com a ferida em 63,2\% (60) dos casos. A assistência dos filhos foi verificada em 20\% (19) dos casos, seguido dos cuidados prestados por cônjuges (9,5\%; 9) e outras pessoas, como cunhados, irmãos, sobrinhos e profissionais de saúde $(7,4 \% ; 7)$.

\section{DISCUSSÃO}

Estudos apontam a maior prevalência de UV em mulheres ${ }^{9-11}$. Porém apesar da maior prevalência no grupo feminino, os estudos não mostram significância estatística entre a ocorrência da ferida e o sexo.

Baixa escolaridade e baixa renda são características socioeconômicas comuns às pessoas com UV em acompanhamento no sistema público de saúde, sendo corroboradas por outras pesquisas no cenário nacional ${ }^{11-13}$

A incidência da UV aumenta com o avançar da idade, ocorrendo com maior frequência no grupo de meia-idade e idosos ${ }^{9-12}$. Devido às alterações fisiológicas inerentes, a exemplo de menor aporte imunológico, diminuição do tônus muscular e maior fragilidade da pele e o acúmulo de comorbidades, o envelhecimento é um fator predisponente para a ocorrência de lesões cutâneas.

A hipertensão foi a comorbidade mais prevalente e sua associação com o diabetes mellitus a combinação mais comum. Este achado é comum a outros estudos em diferentes contextos $^{10,12,13}$. A hipertensão, a dislipidemia e o diabetes mellitus associados à idade avançada favorecem o aparecimento de doença microvascular, com disfunção da função endotelial, levando à diminuição progressiva do fluxo vascular. Esse mecanismo favorece a criação de um microambiente isquêmico para os tecidos, com consequente retardo no reparo tecidual, uma vez que a atividade inflamatória e a síntese tecidual são oxigênio- dependentes ${ }^{10,14}$

Em pessoas com diabetes o processo de cicatrização é lentificado. Alguns mecanismos apontados como fatores importantes para o retardo da cicatrização são: produção excessiva de espécies reativas de oxigênio (ERO), diminuição do óxido nítrico (NO) e diminuição à resposta dos fatores de crescimento. A produção excessiva de ERO é um fator primário que contribui para a deficiência da cicatrização. A redução de NO prejudica a angiogênese e a mobilização de células para o leito da ferida ${ }^{12}$.

Em revisão integrativa realizada por Almeida et al. (2016) foi verificada a influência de fármacos na formação de lesões por pressão, o que pode ser explicado pelas modificações sistêmicas que provocam reações no organismo humano. Nesse sentido, o uso de medicamento pode contribuir no retardo na cicatrização da UV, devido às alterações sistêmicas e efeitos adversos causados pelo uso contínuo.

$\mathrm{O}$ uso de anti-inflamatórios retarda a resposta inflamatória da primeira etapa do processo de cicatrização. Anti-hipertensivos alteram a perfusão tecidual e podem apresentar alguns efeitos adversos, como rash cutâneo e vasoconstrição periférica. Esses medicamentos acrescidos de analgésicos e antibióticos foram descritos por pesquisas como frequentes em pessoas com lesão por pressão ${ }^{12}$.

Neste estudo as úlceras foram localizadas majoritariamente na região distal da perna e região maleolar. Fato comum em outros estudos acerca do tema e já consolidado em literatura ${ }^{9-12}$. Em algumas circunstâncias, a UV pode ocorrer na parte superior da panturrilha ou dos pés, porém nessas situações outras etiologias de úlceras crônicas devem ser excluídas antes de se atribuir a etiologia venosa ${ }^{2,13}$.

A bota de Unna foi a terapia mais utilizada a nível ambulatorial para o tratamento da ferida. Trata-se de bandagem inelástica impregnada com pasta à base óxido de zinco, que auxilia no retorno venoso e na diminuição do edema, ao mesmo tempo em que propicia um ambiente úmido que favorece o processo de cicatrização ${ }^{2,13}$.

Outras coberturas primárias, como alginato de cálcio e hidrofibra com prata são utilizadas para auxiliar no controle do exsudato, sendo as coberturas com prata (hidrofibra e carvão ativado) utilizadas para a prevenção e/ou controle de infecção. $\mathrm{O}$ controle do exsudato favorece a granulação no leito da ferida e evita complicações perilesão, como maceração de bordas e agravamento do eczema, favorecendo a diminuição do tamanho e cicatrização da ferida ${ }^{2,13}$.

Desse modo, foi verificada a adequação do tratamento utilizado no serviço com as recomendações da literatura para o tratamento das UVs, uma vez que a terapia compressiva é considerada o padrão ouro de tratamento para úlceras dessa etiologia ${ }^{1}$. Porém a nível domiciliar é verificada baixa adesão à terapia compressiva por parte dos usuários, principalmente quanto ao uso da faixa elástica e da meia compressiva. 
Vale ressaltar que nos serviços públicos de saúde há grande sazonalidade de produtos e materiais utilizados na assistência à pessoa com ferida, devido aos processos licitatórios e pregões. Neste sentido, a disponibilidade de produtos e coberturas é um fator importante para o seguimento com a terapia tópica adequada, assim como a adesão da pessoa ao tratamento e ao seguimento no serviço.

O perfil de baixa escolaridade e baixa renda apresentado pelas pessoas acompanhadas no serviço público dificultam a aquisição de produtos e coberturas para o tratamento da ferida, levando as mesmas a utilizarem apenas o que está disponível no serviço. Isso pode ser verificado pela pequena quantidade de pessoas que faziam curativo no domicílio com o uso de cobertura primária, assim como os que utilizavam meias ou faixa elástica como terapia compressiva.

Esse fato interfere diretamente na cicatrização da ferida, uma vez que a terapia adequada poderá não estar disponível no serviço de saúde e não estar ao alcance da pessoa com ferida, devido a um baixo poder aquisitivo. Nesse contexto, há que se considerar, ainda, o processo de trabalho dos serviços de saúde, com sobrecarga da assistência pela grande demanda de atendimento. Sendo esses fatores intervenientes que contribuem para a cronificação da ferida, podendo perdurar por anos, assim como para o aumento das recidivas recorrentes.

Pessoas com UV apresentam baixa adesão à terapia compressiva. Fato que pode estar relacionado às falhas no processo de educação em saúde e orientações sobre a terapia compressiva ${ }^{13}$.

Além disso, o fator climático também é um ponto importante que deve ser levado em consideração para a adesão à terapia compressiva. Em ambientes mais quentes o calor contribui para aumentar a sensação térmica, causando desconforto e aumentando a umidade no local que, aliada ao exsudato próprio da ferida, contribui para a ocorrência de maceração dos bordos e complicações perilesão, como as dermatites. Para minimizar esses efeitos seria necessário aumentar a frequência de troca das coberturas, entretanto, isso nem sempre é viável pela baixa disponibilidade de recursos materiais e humanos.

A presença de uma rede de apoio eficaz, como o auxílio de um companheiro(a) ou familiar, configura-se como um importante aliado para o cuidado com as UVs, pois ajuda no enfrentamento de dificuldades e atua como agente facilitador no processo de tratamento, podendo influenciar positivamente na adesão às condutas adequadas ${ }^{3,10}$.
Devido às alterações inerentes ao envelhecimento, como redução da densidade óssea, massa e força muscular, assim como diminuição progressiva da acuidade visual e auditiva, o idoso apresenta maior fragilidade e dificuldades para a realização de algumas atividades diárias ${ }^{13}$. Nesse sentido, a realização do próprio curativo pelo idoso não é uma tarefa tão simples de ser realizada e pode contribuir para o maior risco de desequilibrio e quedas. A presença de um familiar ou cuidador na realização do curativo se faz importante, pois auxilia na melhor execução do procedimento, assim como reduz o risco de quedas e outros acidentes aos quais os idosos estão expostos quando sozinhos.

A adesão ao tratamento é um dos fatores responsáveis pela redução significativa do tempo de cicatrização e diminuição de recidivas. Acredita-se que o principal motivo das recidivas é a não adesão do indivíduo com relação às medidas preventivas (uso de terapia compressiva, elevação de membros inferiores e prática de exercícios regulares) por desconhecimento, dificuldades para assimilar as informações ou falha na orientação ${ }^{13,14}$.

O seguimento com equipe multiprofissional após a cicatrização da úlcera é uma estratégia importante para manutenção da qualidade de vida e prevenção de recidivas. A avaliação periódica das condições da pele, a avaliação de correção cirúrgica das veias incompetentes, assim como atividades de educação em saúde que estimulem a adesão à terapia compressiva e demais ações de autocuidado contribuem para a diminuição da recorrência das úlceras ${ }^{11}$.

O perfil da pessoa com UV atendida pelo Sistema Único de Saúde - SUS - (idade avançada, baixa renda e baixa escolaridade) pode interferir negativamente no processo de cicatrização da ferida devido à maior dificuldade com relação à aprendizagem e compreensão das orientações recebidas. O letramento em saúde, entendido como competência da pessoa em compreender e aplicar a informação em saúde para julgar e tomar decisões no cotidiano sobre os cuidados de saúde, a fim de manter ou melhorar os aspectos de vida ${ }^{14}$, também está relacionado com a capacidade de compreensão das informações recebidas e consequente adesão ao tratamento.

Nesse sentido, é de fundamental importância a atuação do enfermeiro no processo de educação em saúde, identificando o nível de escolaridade e letramento da pessoa com ferida e os demais envolvidos no cuidado para que as orientações acerca do tratamento sejam repassadas de forma adequada ao nível de compreensão e entendimento das pessoas. Desse modo, as chances de sucesso na adesão à terapêutica proposta são aumentadas, com consequente redução no tempo de cicatrização e diminuição da recorrência de feridas. 
No decorrer do estudo, a principal limitação encontrada foi a falta de informação ou registro de alguns itens do impresso da consulta de enfermagem. Também foi verificado que o impresso passou por aprimoramentos durante o período de coleta, sendo evidenciados impressos com presença ou ausência de itens, assim como a formatação. Isso dificultou a obtenção dos dados, resultando na não utilização de algumas informações nesta pesquisa.

Apesar das limitações apresentadas, este estudo torna-se importante na medida em que contribui com difusão do conhecimento acerca do perfil clínico de pessoas com UVs nos diferentes contextos e regiões do Brasil. Proporciona ainda uma reflexão acerca da assistência prestada e efetividade de intervenções implementadas.

\section{CONCLUSÃO}

Conclui-se que o perfil da pessoa com UV integrante deste estudo é compatível com o perfil apresentado por essa população em outros estudos realizados em diferentes contextos e em diferentes regiões do Brasil.

A delimitação do perfil desta população permite o conhecimento sobre o contexto sociocultural ao qual estão inseridos, assim como a reflexão acerca da assistência prestada e efetividade das intervenções implementadas no tratamento da UV.

\section{CONTRIBUIÇÃO DOS AUTORES}

Conceitualização: Oliveira AS e Alexandre SG; Metodologia: Oliveira AS e Alexandre SG; Investigação: Oliveira AS; Correia DL e Vasconcelos KVP; Redação Primeira versão: Oliveira AS e Alexandre SG; Redação Revisão \& Edição: Oliveira AS; Alexandre SG; Silva FAA e Ferreira SL; Aquisição de Financiamento: Oliveira AS; Correia DL; Vasconcelos KVP e Alexandre SG; Recursos: Oliveira AS; Alexandre SG; Correia DL e Vasconcelos KVP; Supervisão: Alexandre SG.

\section{REFERÊNCIAS}

1. Wounds International. Simplifying venous leg ulcer management. Consensus recommendations. Wounds Internacional 2015. [citado 2020 jan 22] Disponível em: https://www.woundsinternational.com/resources/details/ simplifying-venous-leg-ulcer-management-consensusrecommendations

2. Cardoso LV, Godoy JMP, Godoy MFG, Czorny RCN. Terapia compressiva: bota de Unna aplicada a lesões venosas: uma revisão integrativa da literatura. Rev Esc Enferm USP 2018; 52:e03394. https://doi.org/10.1590/S1980$220 \times 2017047503394$

3. Joaquim FL, Silva RMCRA, Garcia-Caro MP, Cruz-Quintana F, Pereira ER. Impact of venous ulcers on patients' quality of life: an integrative review. Rev Bras Enferm 2018 July/ Aug;71(4):2021-9. https://doi.org/10.1590/0034-7167-20170516

4. Silva TG, Vasconcelos APL, Ramos EVC, Farias Neto JP. Avaliação da qualidade de vida de pacientes portadores de feridas crônicas atendidos no ambulatório de cicatrização do Hospital Universitário de Sergipe. R bras Qual Vida 2017 jul/set;9(3):234-46. https://doi.org/10.3895/rbqv.v9n3.6704

5. Wojastyk LDMC, Paula MAB, Prado MNB. Estomaterapia: influências e repercussões na carreira profissional. ESTIMA Braz J Enterostomal Ther 2020;18:e2020. https://doi. org/10.30886/estima.v18.883_PT
6. Borges EL, Ferraz AF, Carvalho DV, Matos SS, Lima VLAN. Prevenção de recidiva de úlcera varicosa: um estudo de coorte. Acta Paul Enferm 2016 jan/fev;29(1):9-16. https:// doi.org/10.1590/1982-0194201600003

7. Teixeira AKS, Silva LF, Marques ADB, Soares CRS. Caracterização de pacientes com úlcera venosa assistidos em ambulatório de estomaterapia de hospital público. ESTIMA Braz J Enterostomal Ther 2018;16:e0318. https:// doi.org/10.30886/estima.v16.346_PT

8. Cruz CC, Caliri MHL, Bernardes RM. Características epidemiológicas e clínicas de pessoas com úlcera venosa atendidas em unidades municipais de saúde. ESTIMA Braz J Enterostomal Ther 2018;16:e1218. https://doi. org/10.30886/estima.v16.496

9. Souza FJ, Aquino JFST, Silva MAG, Oliveira MF, Dantas SRPE. Medidas não invasivas de prevenção da recidiva de úlcera venosa: revisão integrativa. ESTIMA Braz J Enterostomal Ther 2019;17:e1119. https://doi.org/10.30886/estima. v17.713_PT

10. Liberato SMD, Araújo RO, Souza AJG, Marconato AMP, Costa IKF, Torres GV. Adesão ao tratamento de pessoas com úlceras venosas atendidas na atenção primária à saúde. Aquichan 2017;17(2):128-39. https://doi.org/10.5294/ aqui.2017.17.2.2 
11. Oliveira MF, Viana BJF, Matozinhos FP, Silva MMS, Pinto DM, Moreira AD et al. Feridas em membros inferiores em diabéticos e não diabéticos: estudo de sobrevida. Rev Gaúcha Enferm 2019;40:e20180016. https://doi. org/10.1590/1983-1447.2019.20180016

12. Gamba MA, Petri V, Costa MTF. Úlceras venosas, arteriais e mistas. In: Feridas: Prevenção, Causas e Tratamento. Rio de Janeiro: Santos; 2016. p.263-69.
13. Cavalcante VMV, Alexandre SG, Silva FAA, Santiago JCS, Coelho MMF, Avelino BMA et al. Socioeconomic and clinicalepidemiological profile of people attended in an outpatient clinic for complex wounds. Rev Rene 2020;21:e43918. https://doi.org/10.15253/2175-6783.20202143918

14. Almeida AFS, Soares TSB, Abreu RNDC, Mendonça FAC, Guanabara MAO, Sampaio LRL. Influência de fármacos sobre a formação de úlceras por pressão. Rev Enferm Contemp 2016 jan/jun;5(1):118-24. https://doi.org/10.17267/23173378rec.v5i1.681 